\title{
On Spaces of Infinitesimal Motions and Three Dimensional Henneberg Extensions
}

\author{
James Cruickshank
}

Received: 8 August 2013 / Revised: 21 February 2014 / Accepted: 4 March 2014 /

Published online: 29 April 2014

(C) Springer Science+Business Media New York 2014

\begin{abstract}
We investigate certain spaces of infinitesimal motions arising naturally in the rigidity theory of bar and joint frameworks. We prove some structure theorems for these spaces and, as a consequence, are able to deduce some special cases of a long standing conjecture of Graver, Tay and Whiteley concerning Henneberg extensions and generically rigid graphs.
\end{abstract}

Keywords Frameworks $\cdot$ Rigidity $\cdot$ Henneberg moves

Mathematics Subject Classification 52C25

\section{Introduction}

\subsection{Motivation}

In the rigidity theory of bar and joint frameworks the Henneberg extensions play an important role. Let $G=(V, E)$ be a simple finite graph and let $n$ and $k$ be positive integers, and suppose that $X \subset V$ with $|X|=n+k$ and $F \subset E(X)$ with $|F|=k$. We can form a new graph $G^{\prime}$ by deleting all the edges in $F$ and adding a new vertex of degree $n+k$ that is adjacent to all the vertices of $X$. We say that $G^{\prime}$ is an $n$ dimensional Henneberg k-extension of $G$ that is supported on the vertex set $X$ and the edge set $F$. A major stumbling block to a full understanding of the generic rigidity theory of three dimensional bar and joint frameworks is the lack of good sufficient conditions that ensure that a three dimensional Henneberg 2-extension preserves generic rigidity. By

J. Cruickshank $(\bowtie)$

School of Mathematics, Statistics and Applied Mathematics,

National University of Ireland Galway, Galway, Ireland

e-mail: james.cruickshank@nuigalway.ie 
contrast the planar situation is relatively well understood, and we have an extensive theory of planar rigidity stemming largely from Laman's Theorem [7]. Laman's result can be proved by an inductive argument based on two dimensional Henneberg extensions, so it is obviously of interest to see if similar lines of reasoning can be pursued in the three dimensional case.

Given a graph $G$ and vertices $i$ and $j$ of $G$, we say that $i j$ is an implied edge in three dimensions if any flex of a generic three dimensional framework based on $G$ is an infinitesimal isometry of the set of points corresponding to $\{i, j\}$ (see Sect. 2 for the relevant background material). In particular, every edge of $G$ is an implied edge, but there may be implied edges that are not edges of $G$. An implied $K_{4}$ is a set of implied edges of $G$ that form a complete graph on 4 vertices. The following Conjecture of Graver, Tay and Whiteley remains open. For a discussion of this conjecture and many other related problems the reader should consult [4] (wherein it is referred to as the Henneberg Conjecture) or, for a more recent discussion, [5, Conjecture 2.7].

Conjecture 1 Let $G=(V, E)$ be a generically 3-isostatic graph and suppose that $X$ is a subset of $V$ such that $|X|=5$. Let $e$ and $f$ be distinct edges whose vertices are in $X$. Suppose that $G-\{e, f\}$ does not contain any implied $K_{4}$ whose vertices are a subset of $X$. Then the Henneberg extension that removes $e$ and $f$ and adjoins a vertex with neighbour set $X$ results in a generically 3-rigid graph.

A proof of this conjecture would be a significant step towards a better understanding of generic rigidity in 3-space as it would allow us to characterise generically rigid graphs as precisely those that may be obtained from $K_{3}$ by a sequence of allowable Henneberg $k$-extensions, where $k=0,1$ or 2 .

Conjecture 1 has been proved in certain special cases. For example Graver [4, Sect. 5.5] has shown that if $E(X)-\{e, f\}$ contains a 5-cycle then the conjecture is true. Jackson and Owen [6] have shown that if $E(X)$ contains a 3-cycle containing $e$, and $e$ and $f$ are vertex disjoint then the conjecture is true.

In this article we investigate the infinitesimal dynamic properties of rigid frameworks that admit non-rigid Henneberg 2-extensions. More precisely, we should say that the underlying graph admits a 2-extension that is not generically rigid. As an application of our main result, we are able to prove Conjecture 1 in the case where $|E(X)| \geq 7$-generalising the result of Graver mentioned above. Note that, in the case where $e$ and $f$ are vertex disjoint, this result is already implied by the result of Jackson and Owen mentioned above. However, their proof does rely on the vertex disjointness of $e$ and $f$. Our result is therefore logically independent of theirs.

\subsection{Structure of the Paper}

In Sect. 2 we review some of the basic rigidity theory of frameworks - there are no new results in this section. However we present this material in a matrix algebraic setting that is appropriate to our later needs.

In Sects. 3, 4 and 5 we introduce and study the notion of $p$-admissibility. This is the central concept of the paper and essentially captures the infinitesimal dynamic properties of any rigid framework that admits a Henneberg extension that is not generically 
rigid. We make use of a standard matrix identity known as the Sherman-Morrison formula to organise some of the messy polynomial equations that arise when considering the infinitesimal flexes of a framework. The main technical result of the paper is Theorem 15 which provides interesting geometric information about any possible counterexample to Conjecture 1. It is also, we believe, of independent interest as it seems to suggest a non-obvious connection between Euclidean rigidity and affine rigidity in the sense of [3].

Finally in Sect. 6, we apply the results of the previous sections to prove some special cases of Conjecture 1. We also point out a fundamental obstruction to a proof of the general case.

\subsection{Notation}

\subsubsection{Matrices}

We write $\mathbb{R}^{n}$ for the space of $n \times 1$ column vectors with real entries. Throughout this article, we will write $\mathbb{R}^{m \times n}$ for the set of matrices with real entries that have $m$ rows and $n$ columns. For $p \in \mathbb{R}^{m \times n}$, we write $p_{i}$ for the $i$ th column of $p$. We write $\mathbb{1}$ for the column vector all of whose entries are 1 and we write $I$ for the identity matrix. We write $m^{\mathrm{T}}$ for the transpose of the matrix $m$. In particular, for $u, p \in \mathbb{R}^{n}$ the dot product of $u$ and $p$ is $u^{\mathrm{T}} p$.

\subsubsection{Graphs}

In the article graphs are simple undirected graph with no loops or parallel edges. If $G=$ $(V, E)$ is such a graph and $E^{\prime} \subset E$, then $G-E^{\prime}$ denotes the graph obtained by removing all the edges in $E^{\prime}$ from $G$. If $E^{\prime} \subset E$ then $V(E)$ is the set of vertices in $V$ spanned by $E^{\prime}$. If $V^{\prime} \subset V$ then $E\left(V^{\prime}\right)$ is the set of edges in $E$ spanned by $V^{\prime} . K(X)$ denotes the complete graph with vertex set $X, K_{n}$ denotes the complete graph with $n$ vertices and $K_{p, q}$ denotes the complete bipartite graph with vertex parts of order $p$ and $q$.

\subsubsection{Miscellaneous}

We write $\{\{\cdots\}\}$ to indicate a multiset. Statements such as " $P(x)$ is true for almost

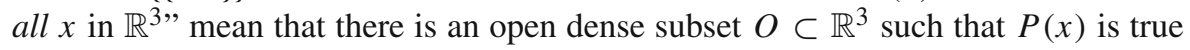
for all $x \in O$.

\section{Point Configurations and Motions}

\subsection{Infinitesimal Isometries of Euclidean Space}

Given a vector field $\xi: \mathbb{R}^{n} \rightarrow \mathbb{R}^{n}$, let $\xi_{x}$ denote the value of the vector field at a point $x \in \mathbb{R}^{n}$. We say that $\xi$ is an infinitesimal isometry of $\mathbb{R}^{n}$ if

$$
\left(\xi_{x}-\xi_{y}\right)^{\mathrm{T}}(x-y)=0
$$


for all $x, y \in \mathbb{R}^{n}$. The set of infinitesimal isometries form a linear subspace of the space of vector fields. Let $\mathcal{I}$ denote this subspace. Let $\mathcal{I}_{0}=\left\{\xi \in \mathcal{I}: \xi_{0}=0\right\}$.

Lemma 2 Given an $n \times n$ skew symmetric matrix a, let $\xi^{a}$ be the vector field on $\mathbb{R}^{n}$ defined by $\xi_{x}^{a}=a x$. The mapping $a \mapsto \xi^{a}$ is a linear isomorphism between the space of skew symmetric matrices and $\mathcal{I}_{0}$.

Proof Let $\xi \in \mathcal{I}_{0}$. Let $a_{\xi}$ be the $n \times n$ matrix whose $i$ th column is $\xi_{e_{j}}$ where $e_{j}$ is the $j$ th standard basis vector of $\mathbb{R}^{n}$. We leave it to the reader to verify that $\xi \mapsto a_{\xi}$ is the inverse of the map defined in the statement.

Lemma 3 For any $\xi \in \mathcal{I}$ there is a unique $t \in \mathbb{R}^{n}$ and a unique skew symmetric $a \in \mathbb{R}^{n \times n}$ such that $\xi_{x}=t+$ ax for all $x \in \mathbb{R}^{n}$.

Proof Let $t=\xi_{0}$ and observe that $t$ is the unique element of $\mathbb{R}^{n}$ such that $\xi-t \in \mathcal{I}_{0}$. Now the lemma follows immediately from Lemma 2.

\subsection{Point Configurations}

Let $n$ be a fixed positive integer. A $k$-point configuration, $p$, in $\mathbb{R}^{n}$ is just an element of $\mathbb{R}^{n \times k}$. We think of $p_{i}$ as the $i$ th point of the configuration.

Given a point configuration $p \in \mathbb{R}^{n \times k}$, an infinitesimal motion of $p$ is a matrix, $u \in \mathbb{R}^{n \times k}$. We think of $u_{i}$ as the velocity vector associated to $p_{i}$. An infinitesimal isometry of $p$ is an infinitesimal motion $u \in \mathbb{R}^{n \times k}$ that satisfies

$$
\left(u_{i}-u_{j}\right)^{\mathrm{T}}\left(p_{i}-p_{j}\right)=0
$$

for all $i, j$. It is common place to refer to an infinitesimal isometry of $p$ as a trivial infinitesimal motion of $p$. Thus, it is important to note the distinction between nontrivial and non-zero in this context.

Observe that any $\xi \in \mathcal{I}$ induces an infinitesimal isometry $u$, of $p$, defined by $u_{i}=\xi_{p_{i}}$. In this way, we can linearly embed $\mathcal{I}$ in $\mathbb{R}^{n \times k}$. We denote the image of $\mathcal{I}$ in $\mathbb{R}^{n \times k}$ by $\mathcal{I}_{p}$. It is easily seen that this embedding is injective if and only if the affine span of $p$ has dimension at least $n-1$. On the other hand, it is also easily seen that any infinitesimal isometry of $p$ is induced by a unique (global) infinitesimal isometry of the affine span of $p$. In particular, any infinitesimal isometry of $p$ is induced by some infinitesimal isometry of $\mathbb{R}^{n}$.

Lemma 4 Given $p \in \mathbb{R}^{n \times k}$ and $u \in \mathbb{R}^{n \times k}$, then $u$ is an infinitesimal isometry of $p$ if and only if there is some skew symmetric matrix $a \in \mathbb{R}^{n \times n}$ and some $t \in \mathbb{R}^{n}$ such that $u=t \mathbb{1}^{\mathrm{T}}+a p$.

Proof As we have just observed, $u$ is an infinitesimal isometry of $p$ if and only there is some infinitesimal isometry $\xi$ of $\mathbb{R}^{n}$ such that $\xi_{p_{i}}=u_{i}$. Now the result follows immediately from Lemma 3.

We say that motions $u$ and $u^{\prime}$ of $p$ are $p$-equivalent if $u-u^{\prime} \in \mathcal{I}_{p}$. We also can extend this notion to subspaces of $\mathbb{R}^{n \times k}$. Thus, linear subspaces $S$ and $S^{\prime}$ of $\mathbb{R}^{n \times k}$ are 
said to to be $p$-equivalent if they have the same dimension and they have the same image under the projections $\mathbb{R}^{n \times k} \rightarrow \mathbb{R}^{n \times k} / \mathcal{I}_{P}$. One readily checks that $S$ and $S^{\prime}$ are $p$-equivalent if and only if there is a linear isomorphism $f: S \rightarrow S^{\prime}$ such that $u-f(u) \in \mathcal{I}_{p}$ for all $u \in S$.

The following notions will be important later on. We say that a vector field $\xi$ on $\mathbb{R}^{n}$ is a linear field if the function $x \mapsto \xi_{x}$ is linear. We say that $\xi$ is an affine field if the function $x \mapsto \xi_{x}$ is an affine linear function. Given a motion $u$ of a point configuration $p$, we say that $u$ is a linear motion, respectively an affine motion, if it is the restriction to $p$ of a linear vector field, respectively an affine vector field. We remark that if $p \in \mathbb{R}^{n \times(n+1)}$ is in general position then any $u \in \mathbb{R}^{n \times(n+1)}$ is an affine motion of $p$. On the other hand the affine motions of a general $q \in \mathbb{R}^{n \times(n+2)}$ form a codimension $n$ affine subspace of the vector space of all motions of $q$.

\subsection{Frameworks}

In this section we review some elementary facts about frameworks. An $n$ dimensional framework is a pair $(G, p)$ where $G$ is a simple undirected graph with vertex set $V$ and edge set $E$ and where $p \in \mathbb{R}^{n \times|V|}$. A $G$-framework is a framework whose underlying graph is $G$. For convenience, we will assume for the moment that $V=\{1, \ldots, k\}$.

An infinitesimal flex of $(G, p)$ is an infinitesimal motion, $u \in \mathbb{R}^{n \times|V|}$, that satisfies

$$
\left(u_{i}-u_{j}\right)^{\mathrm{T}}\left(p_{i}-p_{j}\right)=0
$$

for all $i j \in E$. We say that an infinitesimal flex is trivial if it belongs to $\mathcal{I}_{p}$. A framework is infinitesimally rigid if every infinitesimal flex of the framework is trivial. A framework is isostatic if it is infinitesimally rigid and if the deletion of any edge from $G$ results in a framework that is not infnitesimally rigid.

We will be particularly concerned with frameworks whose underlying point configuration is generic. We say that $p$ is generic if the multiset of its entries is algebraically independent over $\mathbb{Q}$. We observe that generic configurations are in particular in general position. Also, we observe that if $p$ is generic then every submatrix of $p$ has maximal rank.

We say that a graph $G$ is generically $n$-rigid if $(G, p)$ is rigid for any generic configuration $p$ in $\mathbb{R}^{n \times|V|}$. It can be shown that if $(G, p)$ is infinitesimally rigid for some (possibly non-generic) $p \in \mathbb{R}^{n \times|V|}$ then $G$ is generically rigid. On the other hand, it can happen that for a generically rigid graph $G$ there are certain (non-generic) $G$ frameworks that are not infinitesimally rigid. As mentioned in Sect. 1, a fundamental open problem in combinatorial rigidity theory is to find a good characterisation of generically $n$-rigid graphs for $n \geq 3$. A dimension counting argument suggests that $n$ dimensional Henneberg extensions might preserve generic $n$-rigidity. However, as is well known, this is not in general true-see Example 6 below. 


\section{3 p-Admissible Subspaces}

Let $k$ and $n$ be positive integers such that $k \geq n+1$. Suppose that $K_{k, 1}$ is the complete bipartite graph with vertex sets $\{1, \ldots, k\}$ and $\{k+1\}$. For $p \in \mathbb{R}^{n \times k}$ and $x \in \mathbb{R}^{n}$, let $p^{x}=\left(\begin{array}{ll}p & x\end{array}\right)($ Fig. 1$)$.

Definition 5 A linear subspace $S \leq \mathbb{R}^{n \times k}$ will be called $p$-admissible if it has the following properties:

(1) $S \cap \mathcal{I}_{p}=0$.

(2) For almost all $x \in \mathbb{R}^{n}$ there is some non-zero $u \in S$ such that $u$ is the restriction to $p$ of some motion of the framework $\left(K_{k, 1}, p^{x}\right)$.

One may wonder why we restrict to an open dense subset of $\mathbb{R}^{n}$ in the definition of $p$-admissibility. We do this in order to avoid the complications that would arise from degenerate configurations if we had to check the condition for all $x \in \mathbb{R}^{n}$. In all applications of this concept that we have in mind, it is sufficient that condition (2) above be satisifed on an open dense subset.

In order to motivate Definition 5 we consider the following situation. Let $(G, \rho)$ be an isostatic framework in $\mathbb{R}^{n}$ and let $p \in \mathbb{R}^{n \times k}$ be the first $k$ columns of $\rho$. We assume that $V(G)=\{1, \ldots, l\}$ for some $l \geq k$. Let $E^{\prime} \subset E$ be a set of edges of $G$ such that $k=\left|E^{\prime}\right|+n$ and such that $V\left(E^{\prime}\right) \subset\{1, \ldots, k\}$. Since $(G, \rho)$ is isostatic the framework $\left(G-E^{\prime}, \rho\right)$ has a $\left|E^{\prime}\right|$ dimensional space of flexes $\bar{S}$ such that any nontrivial element of $\bar{S}$ restricts to a nontrivial motion of $p$. Thus $\bar{S}$ induces an $\left|E^{\prime}\right|$ dimensional subspace $S$ of $\mathbb{R}^{n \times k}$ such that $S \cap \mathcal{I}_{p}=0$. It is clear that $S$ is $p$-admissible if and only if almost every Henneberg $\left|E^{\prime}\right|$-extension (of the framework) that deletes $E^{\prime}$ and adjoins a vertex adjacent to $\{1, \ldots, k\}$ results in a non-rigid framework. Thus, we see the relevance of understanding $p$-admissible subspaces of $\mathbb{R}^{3 \times 5}$. In particular, any rigid generic framework that admits a 2-extension that is not generically rigid will give rise to a two dimensional $p$-admissible subspace of $\mathbb{R}^{3 \times 5}$ where $p$ is some generic element of $\mathbb{R}^{3 \times 5}$.

Example 6 Let $G$ be the graph obtained by removing a single edge from $K_{5}$ and let $p$ be a generic embedding of the vertex set into $\mathbb{R}^{3}$. It is well known [9, for example] and elementary that a Henneberg 2-extension of $(G, p)$ that removes two edges that are both incident to the same vertex of degree 3 results in a non-rigid framework. See Fig. 2 for an illustration. The framework on the left has a two dimensional space

Fig. 1 A point configuration $p$ and the framework $\left(K_{3,1}, p^{x}\right)$

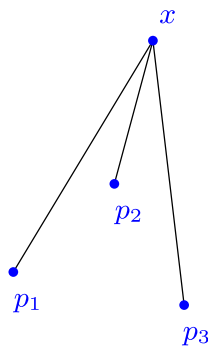


Fig. 2 Notice that the framework on the right is not rigid regardless of where $x$ is placed
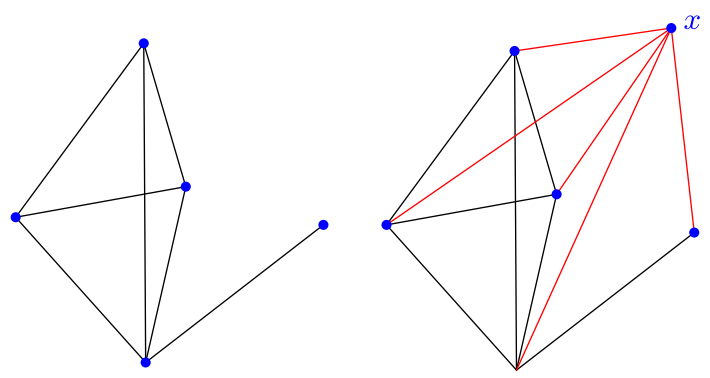

of non-trivial flexes. Clearly at least one non-trivial flex survives in the right hand framework, regardless of the placement of the new vertex $x$. Consideration of this framework leads us to our first example of a $p$-admissible space. Let $V$ be any two dimensional subspace of $\mathbb{R}^{3}$ and let

$$
S=\left\{u \in \mathbb{R}^{3 \times 5}: u_{1} \in V, u_{2}=u_{3}=u_{4}=u_{5}=0\right\} .
$$

Then $S$ is $p$-admissible for any $p \in \mathbb{R}^{3 \times 5}$. Essentially this space is the space of motions that arises after we delete the two edges from the $G$-framework described above. Actually, it is slightly more general than that, as we do not require that $V=\left(p_{1}-p_{i}\right)^{\perp}$ for some $i$.

Indeed, Conjecture 1 is equivalent to the assertion that, for $p \in \mathbb{R}^{3 \times 5}$, the only $p$-admissible subspaces that arise from the deletion of two edges from a generic isostatic framework whose vertices include $p$ are those that are $p$-equivalent to the one described in Example 6. There are however, examples of $p$-admissible spaces that are not equivalent to the one described in Example 6.

Example 7 Let $p \in \mathbb{R}^{3 \times 5}$ be in general position. Fix some constant $k \in \mathbb{R}$ and let

$$
S=\left\{u \in \mathbb{R}^{3 \times 5}: u_{1}^{\mathrm{T}}\left(p_{1}-p_{2}\right)=0, u_{2}=k u_{1}, u_{3}=u_{4}=u_{5}=0\right\} .
$$

Then $S$ is a two dimensional $p$-admissible space. To understand this example, observe that this space of motions arises from the structure shown in the left side of Fig. 3. Of course, this structure is not a generic bar and joint framework, since $p_{1}, p_{2}$ and $y$ are constrained to lie on a single bar. However, we can realise this structure so that $p$ is a generic configuration of five points in $\mathbb{R}^{3}$. It is clear from the illustration in the right hand side of Fig. 3 that the structure will be not be rigid regardless of where $x$ is placed. Thus, one of the non-trivial infinitesimal flexes of the left hand structure survives in the right hand structure. Thus we see that the space of motions of $p$ induced by the left hand structure is indeed $p$-admissible.

One consequence of Example 7 is that any proof of Conjecture 1 must implicity or explicitly demonstrate that this $p$-admissible space cannot arise by deleting two edges from a generic isostatic framework. In fact the situation is even more complicated, as we will show below that there are many other essentially inequivalent examples of $p$-admissible spaces for generic $p \in \mathbb{R}^{3 \times 5}$. 

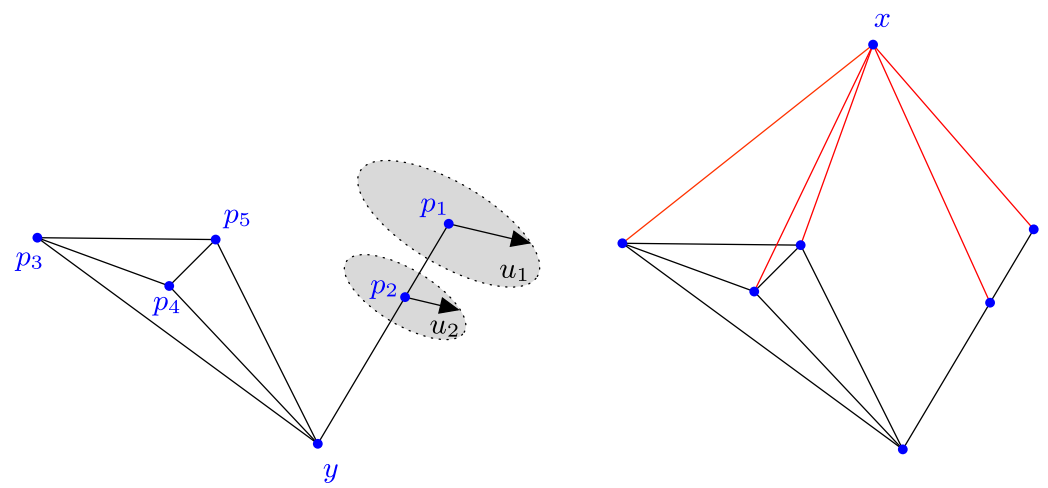

Fig. 3 The structure on the left gives rise to the $p$-admissible space from Example 7. Notice that the structure on the right is not rigid regardless of the placement of $x$

\section{$4 K_{n, 1}$-Frameworks}

Observe that $K_{5,1}$ is the union of two copies of $K_{3,1}$ that have one edge in common. Thus in order to understand flexes of $K_{5,1}$-frameworks we should first understand the flexes of $K_{3,1}$-framework and then consider flexes of two $K_{3,1}$-frameworks with a common edge. In this section we will begin that programme by deriving some basic results concerning flexes of $K_{n, 1}$-frameworks in $\mathbb{R}^{n}$.

Let $q \in \mathbb{R}^{n \times n}$ be a configuration of $n$ points in $\mathbb{R}^{n}$ and let $v \in \mathbb{R}^{n \times n}$ be an infinitesimal motion of $q$. For a square matrix $b$, let $\Delta(b)$ denote the column vector whose entries are the diagonal entries of $b$. For $x \in \mathbb{R}^{n}$ such that $\mathbb{1} x^{\mathrm{T}}-q^{\mathrm{T}}$ is invertible, define

$$
\mathcal{P}(x, q, v)=\left(\mathbb{1} x^{\mathrm{T}}-q^{\mathrm{T}}\right)^{-1}\left(v^{\mathrm{T}} x-\Delta\left(v^{\mathrm{T}} q\right)\right) .
$$

We observe that, if $q$ is an invertible matrix, then $\mathbb{1} x^{\mathrm{T}}-q^{\mathrm{T}}$ is invertible if and only if $x$ does not belong to the affine span of the columns of $q$. Moreover, in this case

$$
\left(\mathbb{1} x^{\mathrm{T}}-q^{\mathrm{T}}\right)^{-1}=-\left(q^{\mathrm{T}}\right)^{-1}\left(I+\frac{\mathbb{1}\left(q^{-1} x\right)^{\mathrm{T}}}{1-\left(q^{-1} x\right)^{\mathrm{T}} \mathbb{1}}\right) .
$$

Identity (4) is sometimes referred to as the Sherman-Morrison formula (see [1] or [8]) and is elementary to verify. The relevance of $\mathcal{P}$ to our discussion is apparent from the following observation. (Recall that, for $x \in \mathbb{R}^{n}, q^{x}=\left(\begin{array}{ll}q & x\end{array}\right)$. $)$

Lemma 8 Suppose that $q$ is an invertible matrix and that $x$ does not belong to the affine span of the rows of $q$. Then $\mathcal{P}(x, q, v)$ is the unique vector such that $(v, \mathcal{P}(x, q, v))$ is a flex of the framework $\left(K_{n, 1}, q^{x}\right)$.

Proof An elementary calculation verifies that

$$
\left(\mathcal{P}(x, q, v)-v_{i}\right)^{\mathrm{T}}\left(x-q_{i}\right)=0
$$

for $i=1,2, \ldots, n$. The uniqueness statement follows from the fact that our hypotheses ensure that the vectors $x-q_{i}, i=1,2, \ldots, n$, are linearly independent. 
Thus, for almost all $x$ and $q$, we have

$$
\mathcal{P}(x, q, v)=-\left(q^{\mathrm{T}}\right)^{-1}\left(I+\frac{\mathbb{1}\left(q^{-1} x\right)^{\mathrm{T}}}{1-\left(q^{-1} x\right)^{\mathrm{T}} \mathbb{1}}\right)\left(v^{\mathrm{T}} x-\Delta\left(v^{\mathrm{T}} q\right)\right) .
$$

In order to understand the flexes of a $K_{5,1}$-framework, we will have to consider solutions of an equation of the form

$$
\mathcal{P}(x, q, v)=\mathcal{P}(x, r, w),
$$

where $q$ and $r$ are three point configurations with a common point and $v$ and $w$ are motions that agree on the common vertex. This leads to rather complicated equations. So we will also consider a certain limit associated to the function $\mathcal{P}$. This argument is somewhat inspired by the viewpoint of coarse geometry - that is that certain geometric problems can be simplified by looking at them from 'far away'. Define

$$
\mathcal{L}(x, q, v)=\lim _{t \rightarrow+\infty} \frac{\mathcal{P}(t x, q, v)}{t} .
$$

Lemma 9 Suppose that $q$ is invertible and that $\left(q^{-1} x\right)^{\mathrm{T}} \mathbb{1} \neq 0$. Then $\mathcal{L}(x, q, v)$ is defined and

$$
\mathcal{L}(x, q, v)=-\left(q^{\mathrm{T}}\right)^{-1}\left(I-\frac{\mathbb{1}\left(q^{-1} x\right)^{\mathrm{T}}}{\left(q^{-1} x\right)^{\mathrm{T}} \mathbb{1}}\right) v^{\mathrm{T}} x .
$$

Proof This follows easily by combining Eq. 5 with the definition of $\mathcal{L}(x, q, v)$.

Note that $\left(q^{-1} x\right)^{\mathrm{T}} \mathbb{1}=0$ if and only if $x$ belongs to the linear subspace that is parallel to the affine span of the columns of $q$.

While the right hand side of Eq. 6 may seem rather complicated still, it is worth noting that the matrix $I-\frac{\mathbb{1}\left(q^{-1} x\right)^{\mathrm{T}}}{\left(q^{-1} x\right)^{\mathrm{T}} \mathbb{1}}$ is just the matrix of the projection parallel to $\mathbb{1}$ onto the orthogonal complement of $\left(q^{-1} x\right)^{\mathrm{T}}$. Thus the geometry of $\mathcal{L}$ is more transparent than that of $\mathcal{P}$.

We also note that, given $x, \mathcal{L}(x, q, v) \in x^{\perp}$ for all $q$ and $v$. Thus, if $\pi$ is a fixed linear mapping on $\mathbb{R}^{n}$ of rank $n-1$, then for almost all $x, \mathcal{L}(x, q, v)$ is determined by $x$ and $\pi(\mathcal{L}(x, q, v))$. By contrast, it is not possible to infer that $\mathcal{P}(x, q, v)$ belongs to any specific $n-1$ dimensional subspace of $\mathbb{R}^{n}$ that depends only on $x$.

\subsection{On $n+1$ Points in $\mathbb{R}^{n}$}

We conclude this section by proving that there are essentially no non-trivial one dimensional $p$-admissible spaces. The proof of this result is instructive as it shows how the algebra of quadratic forms that arise from the functions $\mathcal{L}$ described above can lead to interesting geometric information about infinitesimal flexes of frameworks. This is an idea that will reoccur in later sections, hence one can view Proposition 10 as a warmup for the main technical results that we will see in Sect. 5. 
Proposition 10 Let $p \in \mathbb{R}^{n \times(n+1)}$ be in general position. There is no one dimensional $p$-admissible subspace of $\mathbb{R}^{n \times(n+1)}$.

Proof The case $n=1$ is easy and left to the reader. We assume from now on that $n \geq 2$. Let $q$, respectively $r$, be obtained by deleting the $n$ th, respectively the $(n+1)$ th, column of $p$. Replacing $p$ by a suitable translate if necessary, we may assume that $q$ and $r$ are both invertible. Now our general position hypothesis ensures that $\left(q^{\mathrm{T}}\right)^{-1} \mathbb{1} \neq\left(r^{\mathrm{T}}\right)^{-1} \mathbb{1}$. Moreover, since $r^{-1} q$ is identical to $I$ in its first $n-1$ columns, it is clear that $\left\{\left\{\left(q^{\mathrm{T}}\right)^{-1} \mathbb{1},\left(r^{\mathrm{T}}\right)^{-1} \mathbb{1}\right\}\right\}$ is in fact linearly independent. Suppose that $S=\langle u\rangle$ is a one dimensional $p$-admissible subspace of $\mathbb{R}^{n \times(n+1)}$. Let $v$, respectively $w$, be obtained by deleting the $n$ th, respectively the $(n+1)$ th, columns of $u$. By the definition of $p$-admissibility, we have that $\mathcal{P}(x, q, v)=\mathcal{P}(x, r, w)$ for almost all $x$. By taking a suitable limit we see that $\mathcal{L}(x, q, v)-\mathcal{L}(x, r, w)=0$ for almost all $x$. Therefore

$$
\left(v q^{-1}\right)^{\mathrm{T}} x-\left(w r^{-1}\right)^{\mathrm{T}} x=\left(q^{\mathrm{T}}\right)^{-1} \mathbb{1} \frac{x^{\mathrm{T}}\left(v q^{-1}\right)^{\mathrm{T}} x}{\left(q^{-1} x\right)^{\mathrm{T}} \mathbb{1}}-\left(r^{\mathrm{T}}\right)^{-1} \mathbb{1} \frac{x^{\mathrm{T}}\left(w r^{-1}\right)^{\mathrm{T}} x}{\left(r^{-1} x\right)^{\mathrm{T}} \mathbb{1}}
$$

for almost all $x$. But $\left\{\left\{\left(q^{\mathrm{T}}\right)^{-1} \mathbb{1},\left(r^{\mathrm{T}}\right)^{-1} \mathbb{1}\right\}\right\}$ is linearly independent so there can be no cancellation between the two terms on the right hand side above. Therefore the rational functions

$$
x \mapsto \frac{x^{\mathrm{T}}\left(v q^{-1}\right)^{\mathrm{T}} x}{\left(q^{-1} x\right)^{\mathrm{T}} \mathbb{1}} \text { and } x \mapsto \frac{x^{\mathrm{T}}\left(w r^{-1}\right)^{\mathrm{T}} x}{\left(r^{-1} x\right)^{\mathrm{T}} \mathbb{1}}
$$

must both in fact be linear functions. In other words, $x^{\mathrm{T}}\left(v q^{-1}\right)^{\mathrm{T}} x=x^{\mathrm{T}}\left(q^{-1}\right)^{\mathrm{T}} \mathbb{1}^{\mathrm{T}} x$ for all $x$ and for some $z \in \mathbb{R}^{3}$. Now using the elementary fact that $x^{\mathrm{T}} M x=0$ for all $x$ if and only if $M$ is skew symmetric, it follows that $v=z \mathbb{1}^{\mathrm{T}}+a q$ for some skew symmetric matrix $a$. Thus, by Lemma $4, v$ is an isometry of $q$. Similarly $w$ an isometry of $r$. Now since $\mathcal{P}(x, q, v)-\mathcal{P}(x, r, w)=0$ for all $x$, it follows easily that $u$ is an isometry of $p$, contradicting the fact that $S \cap \mathcal{I}_{p}=0$.

Proposition 10 immediately implies the well known fact (see, for example [4, Chap. 5]) that any Henneberg 1-extension of a generically $n$-isostatic graph is also $n$-isostatic. Indeed most proofs of that fact in the literature essentially amount to proofs of Proposition 10. Of course none of these previous proofs use the $p$-admissibility concept explicitly. Typically some special position argument is used to demonstrate that for any non-trivial motion of $n+1$ points in $\mathbb{R}^{n}$ there is some placement of the point $x$ which will not admit an extension of that flex to the new framework. We have included another proof here for the sake of completeness and also to illustrate that our matrix algebraic viewpoint provides another way to understand some well known geometric results from the literature.

\section{5 -Admissibility for Five Points in $\mathbb{R}^{3}$}

In this section we will use the results of the previous section to analyse two dimensional $p$-admissible spaces where $p$ is a five point configuration in $\mathbb{R}^{3}$. Given that we are 
restricting our attention to such a special situation we find it appropriate to introduce the following special notational conventions. For $p \in \mathbb{R}^{3 \times 5}, q$ is the matrix obtained by deleting the second and third columns of $p$, whereas $r$ is the matrix obtained by deleting the fourth and fifth columns of $p$. Similarly for $u \in \mathbb{R}^{3 \times 5}, v$ is the matrix obtained by deleting the second and third columns of $u$, whereas $w$ is the matrix obtained by deleting the fourth and fifth columns of $u$.

Lemma 11 Let $p, u \in \mathbb{R}^{3 \times 5}$. Then $u$ is a linear motion of $p$ if and only if there is some $m \in \mathbb{R}^{3 \times 3}$ such that $v=m q$ and $w=m r$. In particular, if $q$ is invertible then $u$ is a linear motion of $p$ if and only if $w=v q^{-1} r$.

Proof By definition $u$ is a linear motion of $p$ if and only if there is some $m \in \mathbb{R}^{3 \times 3}$ such that $u_{i}=m p_{i}$.

Lemma 12 Suppose that $u, u^{\prime} \in \mathbb{R}^{3 \times 5}$ are motions of $p \in \mathbb{R}^{3 \times 5}$. Then $u$ and $u^{\prime}$ are $p$-equivalent if and only if there is some skew symmetric matrix $a \in \mathbb{R}^{3 \times 3}$ and some $t \in \mathbb{R}^{3}$ such that $v+a q+t \mathbb{1}^{\mathrm{T}}=v^{\prime}$ and $w+a r+t \mathbb{1}^{\mathrm{T}}=w^{\prime}$

Proof This follows from the definition of $p$-equivalence and Lemma 3.

Now we come to the main technical results of the paper. For the rest of this section we assume that both $q$ and $r$ are invertible. First we recharacterise $p$-admissibility of a subspace $S$ of $\mathbb{R}^{3 \times 5}$.

Proposition 13 Let $S$ be a two dimensional subspace of $\mathbb{R}^{3 \times 5}$ such that $S \cap \mathcal{I}_{p}=0$. Then $S$ is p-admissible if and only if the linear mapping $h_{x}: S \rightarrow \mathbb{R}^{3}$ defined by $h_{x}(u)=\mathcal{P}(x, q, v)-\mathcal{P}(x, r, w)$ has rank at most one for almost all $x \in \mathbb{R}^{3}$.

Proof Observe that the linear mapping $h_{x}$ defined in the statement has rank at most one if and only if there is some non-zero $u \in S$ such that

$$
\mathcal{P}(x, q, v)=\mathcal{P}(x, r, w)
$$

(using the notational convention specified above). But Eq. 7 is true if and only if $(u \mathcal{P}(x, q, v))$ is a flex of $\left(K_{5,1}, p^{x}\right)$.

Proposition 13 allows us to derive an interesting sufficient condition for $p$ admissibility.

Proposition 14 Suppose that $S$ is a two dimensional subspace of $\mathbb{R}^{3 \times 5}$ with the following three properties.

A1. $S \cap \mathcal{I}_{p}=0$.

A2. Every $u \in S$ is a linear motion of $p$.

A3. For all $u \in S$ we have $\left(q^{\mathrm{T}}\right)^{-1} \triangle\left(v^{\mathrm{T}} q\right)=\left(r^{\mathrm{T}}\right)^{-1} \triangle\left(w^{\mathrm{T}} r\right)$.

Then $S$ is $p$-admissible. 
Proof By Lemma 11, $v q^{-1}=w r^{-1}$ for all $u \in S$. Thus, combining Eq. 5 with the definition of $h_{x}$, we see that

$$
h_{x}(u)=\left(\frac{\left(r^{\mathrm{T}}\right)^{-1} \mathbb{1}}{1-\left(r^{-1} x\right)^{\mathrm{T}} \mathbb{1}}-\frac{\left(q^{\mathrm{T}}\right)^{-1} \mathbb{1}}{1-\left(q^{-1} x\right)^{\mathrm{T}} \mathbb{1}}\right)\left(x^{\mathrm{T}}\left(v q^{-1}\right)^{\mathrm{T}} x-\left(q^{-1} x\right)^{\mathrm{T}} \Delta\left(v^{\mathrm{T}} q\right)\right) .
$$

In particular $h_{x}(u)=f_{x}(u) J_{x}$ where $J_{x} \in \mathbb{R}^{3}$ depends only on $p$ and $x$ and $u \mapsto f_{x}(u)$ is a linear functional. Therefore $h_{x}$ has rank at most one for all $x$ for which it is defined and we can conclude, by Proposition 13, that $S$ is $p$-admissible.

Property A3 of Proposition 14 is a rather mysterious looking property, however we will see in Theorem 20 below that it has an interesting consequence for any potential proof of Conjecture 1 . Now we derive a necessary condition for $p$-admissibility.

Theorem 15 Let $p \in \mathbb{R}^{3 \times 5}$ be a five point configuration such that $q$ and $r$ are invertible and such that $\left(r^{-1} q\right)^{\mathrm{T}} \mathbb{1} \neq \mathbb{1}$. Suppose that $S$ is a two dimensional $p$-admissible subspace of $\mathbb{R}^{3 \times 5}$. Then at least one of the following statements is true.

(1) Every element of $S$ is an affine motion of $p$.

(2) $S$ is p-equivalent to a subspace $S^{\prime}$ where $S^{\prime}=E z^{\mathrm{T}}$ for some two dimensional subspace $E$ of $\mathbb{R}^{3}$ and some $z \in \mathbb{R}^{5}$.

Note that hypothetical conditions on $p$ are, in particular, fulfilled by any generic $p \in \mathbb{R}^{3 \times 5}$. The remainder of this section of the paper is devoted to proving Theorem 15. The strategy is as follows. Proposition 13 has allowed us to express $p$-admissibility in terms of $\mathcal{P}(-, q,-)$ and $\mathcal{P}(-, r,-)$. Then a limiting argument (Lemma 16) allows us to obtain a condition on the functions $\mathcal{L}(-, q,-)$ and $\mathcal{L}(-, r,-)$ which is necessary for the space $S$ to be $p$-admissible. We then express this condition in terms of basis elements for the space $S$ and this allows us to reformulate in terms of linear dependence properties of a certain pair of polynomial functions $\mathbb{R}^{2} \rightarrow \mathbb{R}^{2}$. We then derive an elementary characterisation of such pairs (Lemma 17 below). An application of this result allows us to deduce the conclusion of Theorem 15.

Lemma 16 For almostall $x \in \mathbb{R}^{3}$ there is some non-zero $u \in S$ such that $\mathcal{L}(x, q, v)=$ $\mathcal{L}(x, r, w)$

Proof Fix some inner product on the two dimensional real vector space $S$ and let $\tilde{S}$ be the unit sphere with respect to this inner product. Let $t$ be a positive real number. By Proposition 13 there is some $u^{t} \in \tilde{S}$ such that $\mathcal{P}\left(t x, q, v^{t}\right)=\mathcal{P}\left(t x, r, w^{t}\right)$. Since $\tilde{S}$ is compact there is some $u \in \tilde{S}$ and some sequence of real numbers $\left(t_{n}\right)$ such that $t_{n} \rightarrow+\infty$ and $u^{t_{n}} \rightarrow u$ as $n \rightarrow \infty$. Moreover, $\mathcal{P}$ is a rational function and is therefore continuous with respect to the standard topology on its natural domain. Now,

$$
\mathcal{L}(x, q, v)=\lim _{n \rightarrow \infty} \frac{\mathcal{P}\left(t_{n} x, q, v^{t_{n}}\right)}{t_{n}}=\lim _{n \rightarrow \infty} \frac{\mathcal{P}\left(t_{n} x, r, w^{t_{n}}\right)}{t_{n}}=\mathcal{L}(x, r, w) .
$$


Now suppose that $S=\left\langle u^{1}, u^{2}\right\rangle$ where $u^{i} \in \mathbb{R}^{3 \times 5}$. Since $\mathcal{L}(x, q, v)$ is a linear function of $v$, it is clear that there is some non-zero $u \in S$ such that $\mathcal{L}(x, q, v)=$ $\mathcal{L}(x, r, w)$ if and only if

$$
c^{1} \mathcal{L}\left(x, q, v^{1}\right)+c^{2} \mathcal{L}\left(x, q, v^{2}\right)=c^{1} \mathcal{L}\left(x, r, w^{1}\right)+c^{2} \mathcal{L}\left(x, q, w^{2}\right)
$$

for some $\left(c^{1}, c^{2}\right) \neq(0,0)$. Now by rearranging (8) we see that there is some non-zero $u \in S$ such that $\mathcal{L}(x, q, v)=\mathcal{L}(x, r, w)$ if and only if $\left\{\left\{\mathcal{L}\left(x, q, v^{1}\right)-\right.\right.$ $\left.\left.\mathcal{L}\left(x, r, w^{1}\right), \mathcal{L}\left(x, q, v^{2}\right)-\mathcal{L}\left(x, r, w^{2}\right)\right\}\right\}$ is a linearly dependent multiset.

With these observations in mind, and given $p, u^{1}$ and $u^{2}$ as above, we define

$$
f^{i}(x)=\mathcal{L}\left(x, q, v^{i}\right)-\mathcal{L}\left(x, r, w^{i}\right)
$$

for $i=1$, 2. So $f^{i}(x)$ is defined for almost all $x \in \mathbb{R}^{3}$ and $f^{i}(x) \in x^{\perp}$. We have so far shown that if $S$ is $p$-admissible then $\left\{\left\{f^{1}(x), f^{2}(x)\right\}\right\}$ is linearly dependent for almost all $x$. We now turn to an analysis of the functions $f^{i}$.

By Eq. 6

$$
\begin{aligned}
& f^{i}(x)=\left(\left(w^{i} r^{-1}\right)^{\mathrm{T}}-\left(v^{i} q^{-1}\right)^{\mathrm{T}}\right) x \\
& +\left(\frac{\left(q^{\mathrm{T}}\right)^{-1} \mathbb{1}\left(q^{-1} x\right)^{\mathrm{T}}\left(v^{i}\right)^{\mathrm{T}}}{\left(q^{-1} x\right)^{\mathrm{T}} \mathbb{1}}-\frac{\left(r^{\mathrm{T}}\right)^{-1} \mathbb{1}\left(r^{-1} x\right)^{\mathrm{T}}\left(w^{i}\right)^{\mathrm{T}}}{\left(r^{-1} x\right)^{\mathrm{T}} \mathbb{1}}\right) x .
\end{aligned}
$$

This is a rather complicated looking expression, so the next part of the argument (up as far as the statement of Lemma 17) is devoted to transforming $f^{i}$ to a more manageable form.

Suppose that $\pi: \mathbb{R}^{3} \rightarrow \mathbb{R}^{3}$ is some fixed linear mapping (i.e. $3 \times 3$ matrix) of rank two. Since $f^{i}(x) \in x^{\perp}$, we observe that for almost all $x,\left\{\left\{f^{1}(x), f^{2}(x)\right\}\right\}$ is dependent if and only if $\left\{\left\{\pi\left(f^{1}(x)\right), \pi\left(f^{2}(x)\right)\right\}\right\}$ is dependent. Also, we observe that

$$
q_{1}^{\mathrm{T}}\left(\left(w^{i} r^{-1}\right)^{\mathrm{T}}-\left(v^{i} q^{-1}\right)^{\mathrm{T}}\right)=q_{1}^{\mathrm{T}}\left(w^{i} r^{-1}\right)^{\mathrm{T}}-\left(v_{1}^{i}\right)^{\mathrm{T}}=0
$$

since the first columns of $q$ and $r$ are identical and the first columns of $v^{i}$ and $w^{i}$ are identical. With these observations in mind, we choose $\pi$ to be the projection from $\mathbb{R}^{3}$ to $q_{1}^{\perp}$ with kernel $\left\langle\left(q^{\mathrm{T}}\right)^{-1} \mathbb{1}\right\rangle$ and define $\bar{f}^{i}=\pi \circ f^{i}$. Now $q_{1}^{\mathrm{T}}\left(r^{\mathrm{T}}\right)^{-1} \mathbb{1}=1$ since $q_{1}=p_{1}=r_{1}$. Therefore $\pi\left(\left(r^{\mathrm{T}}\right)^{-1} \mathbb{1}\right)=\left(r^{\mathrm{T}}\right)^{-1} \mathbb{1}-\left(q^{\mathrm{T}}\right)^{-1} \mathbb{1} \neq 0$ (by assumption). Let $c=\left(r^{\mathrm{T}}\right)^{-1} \mathbb{1}-\left(q^{\mathrm{T}}\right)^{-1} \mathbb{1}$. The observations above show that

$$
\bar{f}^{i}(x)=\left(w^{i} r^{-1}-v^{i} q^{-1}\right)^{\mathrm{T}} x-\left(\frac{c}{\left(r^{-1} x\right)^{\mathrm{T}} \mathbb{1}}\right) x^{\mathrm{T}}\left(w^{i} r^{-1}\right)^{\mathrm{T}} x .
$$

Moreover, if $S$ is $p$-admissible then $\left\{\left\{\bar{f}^{1}(x), \bar{f}^{2}(x)\right\}\right\}$ is dependent for almost all $x$.

It is clear that replacing $u^{i}$ by $u^{i}+t^{i} \mathbb{1}^{\mathrm{T}}$ leaves the function $f^{i}$ unchangedone can easily verify this algebraically. However the geometric intuition here is that $t^{i} \mathbb{1}^{\mathrm{T}}$ represents an infinitesimal translation of a particular point configuration. But infinitesimal translations vanish in the limit that occurs in the definition of $\mathcal{L}$ and therefore contribute nothing to $f^{i}$. Now we will choose a particular $t^{i}$ as follows: First 
choose some $d \in \mathbb{R}^{3}$ so that $\{c, d\}$ is a basis for $q_{1}^{\perp}$. By replacing $u^{i}$ by $u^{i}+t^{i} \mathbb{1}^{\mathrm{T}}$ for an appropriate $t^{i} \in \mathbb{R}^{3}$ we can arrange that the column space of the matrix $w^{i} r^{-1}-v^{i} q^{-1}$ is spanned by $d$.

In other words

$$
\left(w^{i} r^{-1}-v^{i} q^{-1}\right)^{\mathrm{T}}=d\left(k^{i}\right)^{\mathrm{T}}
$$

for some $k^{i} \in \mathbb{R}^{3}$. So

$$
\bar{f}^{i}(x)=d\left(k^{i}\right)^{\mathrm{T}} x-\left(\frac{c}{\left(r^{-1} x\right)^{\mathrm{T}} \mathbb{1}}\right) x^{\mathrm{T}}\left(w^{i} r^{-1}\right)^{\mathrm{T}} x .
$$

Now it is clear that $\bar{f}^{i}$ is a rational homogeneous function of degree 1 , so $\left\{\left\{\bar{f}^{1}(x), \bar{f}^{2}(x)\right\}\right\}$ is linearly dependent for almost all $x$ if and only if it is linearly dependent for all $x$ such that $\left(r^{-1} x\right)^{\mathrm{T}} \mathbb{1}=1$. Thus, define $g^{i}:\left\{x:\left(r^{-1} x\right)^{\mathrm{T}} \mathbb{1}=1\right\} \rightarrow\left(q_{1}\right)^{\perp}$ by

$$
g^{i}(x)=d\left(k^{i}\right)^{\mathrm{T}} x-c\left(x^{\mathrm{T}}\left(w^{i} r^{-1}\right)^{\mathrm{T}} x\right) .
$$

By choosing bases, we can think of $g^{i}$ as a polynomial function from $\mathbb{R}^{2} \rightarrow \mathbb{R}^{2}$ where the first coordinate function is affine linear and the second coordinate function is affine quadratic. Moreover, if $S$ is $p$-admissible, then $\left\{\left\{g^{1}(x), g^{2}(x)\right\}\right\}$ is linearly dependent for all $x$ such that $x r^{-1} \mathbb{1}=1$.

Lemma 17 For $i=1,2$ let $h_{i}: \mathbb{R}^{n} \rightarrow \mathbb{R}^{2}$ be of the form $h_{i}(z)=\left(l_{i}(z), q_{i}(z)\right)$ where $l_{i}: \mathbb{R}^{n} \rightarrow \mathbb{R}$ is affine linear and $q_{i}: \mathbb{R}^{n} \rightarrow \mathbb{R}$ is affine quadratic. Then $\left\{\left\{h_{1}(z), h_{2}(z)\right\}\right\}$ is linearly dependent for almost all $z \in \mathbb{R}^{n}$ if and only if at least one of the following conditions is satisfied.

(1) There exist constants $r, s \in \mathbb{R}$ such that $(r, s) \neq(0,0)$ and $r h_{1}+s h_{2} \equiv 0$. (i.e. $\left\{\left\{h_{1}, h_{2}\right\}\right\}$ is linearly dependent over $\left.\mathbb{R}\right)$.

(2) $l_{1} \equiv l_{2} \equiv 0$.

(3) There exists some affine linear function $m: \mathbb{R}^{n} \rightarrow \mathbb{R}$ such that $q_{i} \equiv m l_{i}$ for $i=1,2$.

Proof The "if" direction is trivially easy to verify. Now assume that $\left\{\left\{h_{1}(z), h_{2}(z)\right\}\right\}$ is linearly dependent for all $z \in \mathbb{R}^{n}$. So $l_{1}(z) q_{2}(z)=l_{2}(z) q_{1}(z)$ for all $z \in \mathbb{R}^{n}$. In other words

$$
l_{1} q_{2} \equiv l_{2} q_{1} .
$$

If $l_{1} \equiv 0$ then either $l_{2} \equiv 0$ or $q_{1} \equiv 0$. Thus, either (1) or (2) is true if $l_{1} \equiv 0$. Similarly, either (1) or (2) is true if $l_{2} \equiv 0$. Thus from now on we assume that $l_{1} \not \equiv 0$ and $l_{2} \not \equiv 0$. But $\mathbb{R}\left[X_{1}, \ldots, X_{n}\right]$ is a unique factorisation domain. Therefore Eq. 13 implies that there are two possibilities. Either $l_{1} \equiv \alpha l_{2}$ for some non-zero constant $\alpha \in \mathbb{R}$. This leads to case (1) of the statment. Or $l_{1} \mid q_{1}$ and $l_{2} \mid q_{2}$. This leads to case (3) of the statement.

Now we apply Lemma 17 to the functions $g^{i}, i=1,2$ and we conclude that if $S$ is $p$-admissible then at least one of the following conditions must be satisfied.

$\left(1^{\prime}\right) \quad\left\{\left\{g^{1}, g^{2}\right\}\right\}$ is linearly dependent over $\mathbb{R}$.

$\left(2^{\prime}\right) \quad\left(k^{1}\right)^{\mathrm{T}} x=\left(k^{2}\right)^{\mathrm{T}} x=0$ for all $x$ such that $\left(r^{-1} x\right)^{\mathrm{T}} \mathbb{1}=1$. 
$\left(3^{\prime}\right)$ There is some affine linear function $m:\left\{x:\left(r^{-1} x\right)^{\mathrm{T}} \mathbb{1}=1\right\} \rightarrow \mathbb{R}$ such that $x^{\mathrm{T}}\left(w^{i} r^{-1}\right)^{\mathrm{T}} x=m(x)\left(k^{i}\right)^{\mathrm{T}} x$ for all $x$ such that $\left(r^{-1} x\right)^{\mathrm{T}} \mathbb{1}=1$.

Before proceeding we remind the reader of the following elementary fact: Given $m, m^{\prime} \in \mathbb{R}^{3 \times 3}$, then $x^{\mathrm{T}} m x=x^{\mathrm{T}} m^{\prime} x$ for almost all $x \in \mathbb{R}^{3}$ if and only if $m-m^{\prime}$ is a skew symmetric matrix. Now suppose that $\left(1^{\prime}\right)$ is true. This is equivalent to saying that there exists some $\left(\alpha^{1}, \alpha^{2}\right) \neq(0,0)$ such that

$$
\alpha^{1}\left(w^{1} r^{-1}-v^{1} q^{-1}\right)^{\mathrm{T}}+\alpha^{2}\left(w^{2} r^{-1}-v^{2} q^{-1}\right)^{\mathrm{T}}=0
$$

and

$$
\alpha^{1}\left(\left(w^{1} r^{-1}\right)^{\mathrm{T}}+w^{1} r^{-1}\right)+\alpha^{2}\left(\left(w^{2} r^{-1}\right)^{\mathrm{T}}+w^{2} r^{-1}\right)=0
$$

Now Eq. 15 implies that $\left(\alpha^{1} w^{1}+\alpha^{2} w^{2}\right) r^{-1}$ is skew symmetric. Transposing Eq. 14 and rearranging, we see that $\left(\alpha^{1} v^{1}+\alpha^{2} v^{2}\right) q^{-1}=\left(\alpha^{1} w^{1}+\alpha^{2} w^{2}\right) r^{-1}$. Thus we see that if $\left(1^{\prime}\right)$ is true then $\alpha^{1} u^{1}+\alpha^{2} u^{2}$ is a non-zero infinitesimal isometry of $p$ which contradicts our assumption that $S \cap \mathcal{I}_{p}=0$. Under the assumptions of Theorem 15 we conclude that $\left(1^{\prime}\right)$ cannot be true.

Suppose that $\left(2^{\prime}\right)$ is true. So $\left(w^{i} r^{-1}\right)^{\mathrm{T}}-\left(v^{i} q^{-1}\right)^{\mathrm{T}}=0$ for $i=1,2$. Therefore, by Lemma $11, u^{i}$ is a linear motion of $p$ for $i=1,2$. It follows easily from this that every element of $S$ is a linear motion of $p$. Remembering that we replaced $u^{i}$ by $u^{i}+t^{i} \mathbb{1}^{\mathrm{T}}$ earlier in the argument, we conclude that every element of our original $S$ is in fact an affine motion of $p$.

Finally, suppose that $\left(3^{\prime}\right)$ is true. Then $m(x)=x^{\mathrm{T}} l$ for some $l \in \mathbb{R}^{3}$. Therefore $x^{\mathrm{T}}\left(w^{i} r^{-1}\right)^{\mathrm{T}} x=x^{\mathrm{T}} l\left(k^{i}\right)^{\mathrm{T}} x$ for almost all $x$. As remarked above this implies that $\left(w^{i} r^{-1}\right)^{\mathrm{T}}=l\left(k^{i}\right)^{\mathrm{T}}+a^{i}$ for some skew symmetric $a^{i} \in \mathbb{R}^{3 \times 3}$. Combining this this with Eq. 11, we see that $\left(v^{i} q^{-1}\right)^{\mathrm{T}}=(l-d)\left(k^{i}\right)^{\mathrm{T}}+a^{i}$. Therefore we have

$$
\begin{aligned}
v^{i}+a^{i} q & =k^{i}(l-d)^{\mathrm{T}} q, \\
w^{i}+a^{i} r & =k^{i} l^{\mathrm{T}} r .
\end{aligned}
$$

Now let $z \in \mathbb{R}^{5}$ be the unique vector such that deleting the second and third rows yields $q^{\mathrm{T}}(l-d)$, whereas deleting the fourth and fifth rows of $z$ yields $r^{\mathrm{T}} l$ (this makes sense since $q_{1}^{\mathrm{T}} d=0$ and $\left.r_{1}=q_{1}\right)$. Let $E$ be the subspace of $\mathbb{R}^{3}$ that is spanned by $\left\{k^{1}, k^{2}\right\}$. Then Lemma 12 and Eqs. 16 and 17 imply that $S$ is $p$-equivalent to $E z^{\mathrm{T}}$. This completes the proof of Theorem 15 .

\section{Applications to Rigidity Theory}

The remainder of the paper will be concerned with applications of the results of Sects. 3 , 4 and 5 to the rigidity theory of frameworks.

Lemma 18 Let $p \in \mathbb{R}^{3 \times 5}$ be generic and let $S$ be a two dimensional $p$-admissible subspace of $\mathbb{R}^{3 \times 5}$. Suppose that there is some three point subconfiguration of $p$ such that that for every $u \in S, u$ restricts to a space of isometries of that three point configuration. Then, up to a possible permutation of the points, $S$ is p-equivalent to one of the spaces described in Example 6 or Example 7. 
Proof Using the notational convention of Sect.5, we may assume without loss of generality that $w=0$ for all $u \in S$. So $\mathcal{L}(x, r, w)=0$ for all $x$. Therefore by Lemma 16 the linear mapping $u \mapsto \mathcal{L}(x, q, v)$ has rank at most one for almost all $x$. Now

$$
\mathcal{L}(x, q, v)=-\left(q^{\mathrm{T}}\right)^{-1}\left(I-\frac{\mathbb{1}\left(q^{-1} x\right)^{\mathrm{T}}}{\left(q^{-1} x\right)^{\mathrm{T}} \mathbb{1}}\right) v^{\mathrm{T}} x .
$$

But the projection matrix

$$
I-\frac{\mathbb{1}\left(q^{-1} x\right)^{\mathrm{T}}}{\left(q^{-1} x\right)^{\mathrm{T}} \mathbb{1}}
$$

has kernel $\langle\mathbb{1}\rangle$ and the first row of $v^{\mathrm{T}}$ is zero. Therefore we conclude that the linear mapping $u \mapsto v^{\mathrm{T}} x$ has rank at most one for almost all $x \in \mathbb{R}^{3}$. It follows easily that $S=E z^{\mathrm{T}}$ where $E$ is a two dimensional subspace of $\mathbb{R}^{3}$ and $z^{\mathrm{T}}=(0,0,0, k, 1)$ for some constant $k$ (here we may have to permute vertices 4 and 5 to ensure that the fifth entry of $z^{\mathrm{T}}$ is non-zero). If $k=0$ then $S$ is $p$-equivalent to one of the spaces described in Example 6. It remains to show that if $k \neq 0$ then we must have $E=\left(p_{5}-p_{4}\right)^{\perp}$. Now, since $S=E z^{\perp}$ is $p$-admissible and since $\mathcal{P}(x, r, w)=0$ for almost all $x$ and all $u \in S$, it follows from Definition 5 that for almost all $x$, there is some non-zero $v^{x} \in E$ such that

$$
\begin{aligned}
& \left(v^{x}\right)^{\mathrm{T}}\left(x-p_{4}\right)=0, \\
& \left(v^{x}\right)^{\mathrm{T}}\left(x-p_{5}\right)=0 .
\end{aligned}
$$

Subtracting (19) from (18) yields $v^{x} \in E \cap\left(p_{5}-p_{4}\right)^{\perp}$. If this space has dimension at most one, then it clearly follows that $v^{x}=0$ for almost all $x$ which contradicts the fact that $v^{x} \neq 0$ for almost all $x$. Therefore $\operatorname{dim}\left(E \cap\left(p_{5}-p_{4}\right)^{\perp}\right)=2$ as required.

We can use this to prove a special case of Conjecture 1 . We introduce the following notation for the rest of the section. $G=(V, E)$ is a generically 3-isostatic graph, $X=\{1,2,3,4,5\} \subset V,\{e, f\} \subset E(X)$ and $G^{\prime}$ is the 2-extension of $G$ supported on vertex set $X$ and edge set $\{e, f\}$. Moreover, given a framework $(G, \rho)$, let $p \in \mathbb{R}^{3 \times 5}$ be defined by $p_{i}=\rho_{i}$ for $i \in X$.

Corollary 19 Suppose that $G-\{e, f\}$ contains an implied subgraph $H$ that is isomorphic to the graph shown below

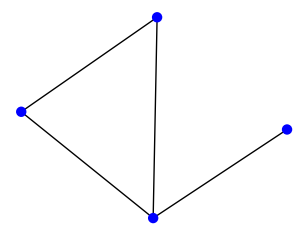

and where the vertices of $H$ are in $X$. Then either $G-\{e, f\}$ contains an implied $K_{4}$ whose vertices are in $X$, or $G^{\prime}$ is generically 3-rigid. 
Proof Let $(G, \rho)$ be a generic framework. Suppose that $G^{\prime}$ is not generically rigid. Then the non-trivial flexes of $(G-\{e, f\}, \rho)$ induce a $p$-admissible subspace of $\mathbb{R}^{3 \times 5}$. Since $H$ contains a triangle, we can apply Lemma 18 to conclude that this space is $p$-equivalent to either Example 6 or 7 . In the first case, it is clear that $G-\{e, f\}$ contains an implied $K_{4}$. So it suffices to show that the space from Example 7 cannot arise from this framework. However this clearly follows from the genericity of $p$ and the constraint imposed by the fourth edge of $H$.

Our next result illustrates a fundamental difficulty that must be resolved directly or indirectly by any possible proof of Conjecture 1 .

Theorem 20 For any generic $p \in \mathbb{R}^{3 \times 5}$ there is a two dimensional $p$-admissible subspace $S$ of $\mathbb{R}^{3 \times 5}$ such that $S$ does not restrict to a space of isometries on any three point subconfiguration of $p$.

Proof In fact we will show that there are infinitely many such subspaces. The proof essentially amounts to a dimension count based on Proposition 14 . Let $L$ be the linear subspace of $\mathbb{R}^{3 \times 5}$ consisting of the linear motions of $p$. Clearly, $L$ has dimension 9 - it is isomorphic to $\mathbb{R}^{3 \times 3}$. Now consider the equation

$$
\left(q^{\mathrm{T}}\right)^{-1} \triangle\left(v^{\mathrm{T}} q\right)=\left(r^{\mathrm{T}}\right)^{-1} \triangle\left(w^{\mathrm{T}} r\right)
$$

as linear constraint on $L$. This constraint has rank at most two, since $q_{1}=r_{1}$ and $v_{1}=w_{1}$. Let $R$ be the subspace of $L$ consisting of solutions of Eq. 20. The dimension of $R$ is at least 7 and $R \cap \mathcal{I}_{p}$ is a three dimensional linear subspace of $R$. Proposition 14 implies that any two dimensional subspace of $R$ that intersects $\mathcal{I}_{p}$ trivially is in fact $p$-admissible. We also observe that two dimensional linear subspaces $S$ and $S^{\prime}$ of $R$ are $p$-equivalent if and only they project to the same subspace of $R /\left(R \cap \mathcal{I}_{p}\right)$. Therefore there is a one to one correspondence between the set of $p$-equivalence classes of two dimensional $p$-admissible subspaces of $R$ and the set of two dimensional linear subspaces of $R /\left(R \cap \mathcal{I}_{p}\right)$. This latter set forms a Grassman manifold $M$ of dimension at least 4 , since the dimension of $R /\left(R \cap \mathcal{I}_{p}\right)$ is at least 4 .

Now suppose that $S$ is one of the $p$-admissible spaces constructed in Example 6 or 7 . It is easy to see that for a given $p$ there are only finitely many such spaces that consist of affine motions. We also note that if $S$ and $S^{\prime}$ are $p$-equivalent spaces and $S$ consists of affine motions, then $S^{\prime}$ consists of affine motions. This follows from the fact that every trivial motion of $p$ is also an affine motion. Therefore there must be many elements of $M$ that are not $p$-equivalent to either Example 6 or 7. By Lemma 18 this means that there are many elements of $M$ that do not restrict to spaces of isometries for any three point subset of $p$.

Contrast this situation with Proposition 10 which tells us that there are no one dimensional $p$-admissible spaces for any generic $p \in \mathbb{R}^{n \times(n+1)}$. As we observed after that proposition, this leads to an immediate understanding of Henneberg 1-extensions. On the other hand any proof of Conjecture 1 must somehow (directly or indirectly) demonstrate that the motions whose existence is asserted by Theorem 20 cannot arise by deleting two edges from a generic framework. Even if we were able to do this, 

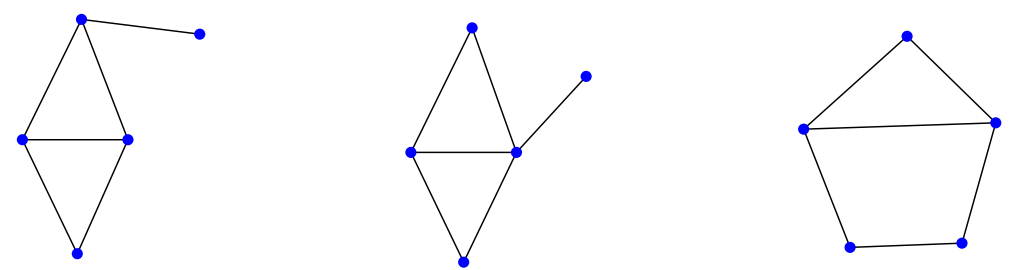

Fig. 4 The three possibilities for the graph $H$ from the proof of Lemma 21

we would still face the difficulty of showing that the $p$-admissible space of Example 7 cannot arise from deleting two edges from a generic isostatic framework. As far as the author is aware, even that special case remains open. Thus Theorem 20 and Example 7 identify a fundamental difficulty with Conjecture 1 that does not arise for the corresponding question about Henneberg 1-extensions. In particular, it suggests that the typical 'special position' type arguments, that can be used in the planar case to show that certain Henneberg extensions preserve generic rigidity, may not be as fruitful in the case of 2-extensions in three dimensional space.

Despite the somewhat negative observations above, we can obtain some positive new partial results toward Conjecture 1.

Lemma 21 Suppose that $S$ is a two dimensional space of affine motions of a generic $p \in \mathbb{R}^{3 \times 5}$ and suppose that $S$ restricts to a space of isometries on each of five distinct edges of $K_{5}$. Then there is some non-zero $u \in S$ that is an infinitesimal isometry of $p$.

Proof The proof is a variation of the standard 'conic at infinity' argument (see [2, Proposition 4.2], for example). First we observe that there are six different isomorphism types of five edge graphs on five vertices. One readily checks that for each of these six graphs it is possible to add one edge so that the resulting graph is isomorphic to one of the three graphs shown in Fig. 4. Now since $S$ is two dimensional it is clear that for any edge $e$ of $K_{5}$ there is some non-zero $u \in S$ such that $u$ is an isometry on $e$. Thus we we can find some non-zero $u \in S$ such that $u$ is a flex of an $H$-framework, where $H$ is isomorphic to one of the three graphs shown in Fig. 4.

Since $u$ is affine, we have $u_{i}=m p_{i}+b$ for some $m \in \mathbb{R}^{3 \times 3}$ and $b \in \mathbb{R}^{3}$. Therefore

$$
\left(p_{i}-p_{j}\right)^{\mathrm{T}} m\left(p_{i}-p_{j}\right)=0
$$

for all $i j$ in $H$. In other words, the six edges of a generic $H$-framework lie on the conic at infinity associated to the matrix $m$. Now we show that this cannot happen if the conic is non-trivial. This follows from the observation that since $H$ contains a triangle of edges, any such conic must be degenerate (i.e. a pair of lines at infinity). Thus in the two cases where $H$ has a pair of triangles, the remaining edge will not (generically) lie on either of the corresponding lines at infinity. In the other case $H$ consists of a triangle and three edges that do not form a triangle. These latter three edges will not generically lie on a single line at infinity, nor will any of them lie on the line at infinity correponding to the triangle. Therefore Eq. 21 must in fact be a trivial quadratic equation. In other words, $m$ is skew symmetric and it follows that $u$ is a trivial motion of $p$. 
Theorem 22 Suppose that $|E(X)| \geq 7$. Then either $G-\{e, f\}$ contains an implied $K_{4}$ whose vertices are in $X$, or $G^{\prime}$ is generically 3-rigid.

Proof Suppose that $G^{\prime}$ is not generically 3-rigid and let $(G, \rho)$ be a generic framework. So $(G-\{e, f\}, \rho)$ has a two dimensional space of flexes that induces a $p$-admissible subspace, $S$, of $\mathbb{R}^{3 \times 5}$. Suppose that $S$ consists of affine motions of $p$. Since $\operatorname{dim}(S)=2$, there is some non-zero $u \in S$ that is non-trivial on $p$ that restricts to an isometry of 6 edges of $K(X)$. By Lemma 21 some non-zero element of $S$ is a trivial motion of $p$ which contradicts the fact that $S$ is $p$-admissible. Therefore by Theorem 15 , we can assume that $S=E z^{\mathrm{T}}$ where $E \leq \mathbb{R}^{3}$ has dimension two and $z \in \mathbb{R}^{5}$. Now suppose that $z_{1}=z_{2}=z_{3}$. Then $S$ restricts to a space of isometries of $\left\{p_{1}, p_{2}, p_{3}\right\}$. But $S$ restricts to a space of isometries of at least two edges of $E(X)-E(\{1,2,3\})$. Therefore, by Corollary 19 we conclude that either $G-\{e, f\}$ contains an implied $K_{4}$ with vertices in $X$ or $G^{\prime}$ is generically rigid. So, from now on we can assume that in the multiset $\left\{\left\{z_{1}, z_{2}, z_{3}, z_{4}, z_{5}\right\}\right\}$, no value occurs with multiplicity greater than two. We will show that this assumption leads to a contradiction. Up to a permutation, there are three cases to consider:

(1) $z_{1}, z_{2}, z_{3}$ are pairwise distinct, $z_{4}=z_{1}$ and $z_{5}=z_{2}$.

(2) $z_{1}, z_{2}, z_{3}, z_{4}$ are pairwise distinct and $z_{5}=z_{1}$.

(3) $z_{1}, z_{2}, z_{3}, z_{4}, z_{5}$ are pairwise distinct.

We will give details only for first of these cases. So suppose that $z_{1}, z_{2}, z_{3}$ are pairwise distinct, and that $z_{4}=z_{1}$ and $z_{5}=z_{2}$. Since $\operatorname{dim}(S)=2$ we can find a non-zero element $c z^{\mathrm{T}} \in S, c \in E$, that is an isomtery of six edges of $K(X)$. But $c z^{\mathrm{T}}$ is an isometry of the edge $i j$ if and only if $\left(z_{i}-z_{j}\right) c^{\mathrm{T}}\left(p_{i}-p_{j}\right)=0$. Now, under our working assumption, $z_{i}-z_{j}=0$ if and only if $i j=14$ or $i j=25$. Therefore there are at least four of the six edges for which $z_{i}-z_{i} \neq 0$. So there are at least four edges for which $c^{\mathrm{T}}\left(p_{i}-p_{j}\right)=0$. But since $p$ is generic, it follows easily that $c=0$ which contradicts the fact that $c z^{\mathrm{T}} \neq 0$. The other two cases can be dealt with in a similar way.

Theorem 22 generalises the result of Graver mentioned in the introduction. In his result, it is required that five of the edges in $E(X)-\{e, f\}$ form a cycle.

Acknowledgments The author would like to thank Bob Connelly for some very helpful conversations on this topic and in particular for pointing out the application of the conic at infinity argument in the proof of Theorem 22. The author also expresses his thanks to Bill Jackson and John Owen for allowing him access to their unpublished manuscript [6]. Thanks are also due to one of the referees for several helpful suggestions regarding the exposition and structure of the paper. Finally I dedicate this article to my daughter Lily whose wakeful nights afforded me lots of time to think about Henneberg extensions.

\section{References}

1. Bartlett, M.S.: An inverse matrix adjustment arising in discriminant analysis. Ann. Math. Stat. 22, 107-111 (1951)

2. Connelly, R.: Generic global rigidity. Discrete Comput. Geom. 33(4), 549-563 (2005)

3. Gortler, S.J., Gotsman, C., Liu, L., Thurston, D.: On affine rigidity (2010). http://arxiv.org/pdf/1011. $5553 \mathrm{v} 1$ 
4. Graver, J., Servatius, B., Servatius, H.: Combinatorial Rigidity. Graduate Studies in Mathematics, vol. 2. American Mathematical Society, Providence, RI (1993)

5. Jackson, B., Jordán, T.: On the rank function of the 3-dimensional rigidity matroid. Int. J. Comput. Geom. Appl. 16(5-6), 415-429 (2006)

6. Jackson, B., Owen, J.: Notes on Henneberg Moves. Unpublished Manuscript

7. Laman, G.: On graphs and rigidity of plane skeletal structures. J. Eng. Math. 4, 331-340 (1970)

8. Sherman, J., Morrison, W.J.: Adjustment of an inverse matrix corresponding to a change in one element of a given matrix. Ann. Math. Stat. 21, 124-127 (1950)

9. Tay, T.-S., Whiteley, W.: Generating isostatic frameworks. Struct. Topol. 11, 21-69 (1985) 